\title{
Squeeze-film damping of flexible microcantilevers at low ambient pressures: theory and experiment
}

\author{
Jin Woo Lee ${ }^{1}$, Ryan Tung ${ }^{2}$, Arvind Raman ${ }^{2}$, Hartono Sumali $^{3}$ and \\ John P Sullivan ${ }^{3}$ \\ ${ }^{1}$ Division of Mechanical Engineering, Ajou University, San 5 Woncheon-Dong, YeongTong-Gu, Suwon \\ 443-749, Republic of Korea \\ ${ }^{2}$ School of Mechanical Engineering, Birck Nanotechnology Center, Purdue University, West Lafayette, \\ IN 47907, USA \\ ${ }^{3}$ Sandia National Laboratories, MS 1070, PO Box 5800, Albuquerque, NM 87185, USA \\ E-mail: raman@purdue.edu
}

Received 11 March 2009, in final form 12 July 2009

Published 24 September 2009

Online at stacks.iop.org/JMM/19/105029

\begin{abstract}
An improved theoretical approach is proposed to predict the dynamic behavior of long, slender and flexible microcantilevers affected by squeeze-film damping at low ambient pressures. Our approach extends recent continuum gas damping models (Veijola $2004 \mathrm{~J}$. Micromech.

Microeng. 14 1109-18, Gallis and Torczynski 2004 J. Microelectromech. Syst. 13 653-9),

which were originally derived for a rigid oscillating plate near a wall, to flexible

microcantilevers for calculating and predicting squeeze-film damping ratios of higher order bending modes at reduced ambient pressures. Theoretical frequency response functions are derived for a flexible microcantilever beam excited both inertially and via external forcing. Experiments performed carefully at controlled gas pressures are used to validate our theoretical approach over five orders of the Knudsen number. In addition, we investigate the relative importance of theoretical assumptions made in the Reynolds-equation-based approach for flexible microelectromechanical systems.
\end{abstract}

(Some figures in this article are in colour only in the electronic version)

\section{Introduction}

Squeeze-film gas damping is a major determinant of the dynamic behavior of microelectromechanical systems (MEMS), especially at low ambient pressures. Gas damping strongly influences the mechanical quality factors of microfabricated resonators and the switching time, impact velocity and bounce of contacting MEMS such as radio frequency (RF) switches [1-4]. To predict the dynamic behavior of an ohmic contact RF MEMS, Guo et al [3] used the finite difference method to present a 3D nonlinear dynamic model covering various aspects of a real switch such as complicated geometry, non-uniform squeeze-film damping and electrostatic actuation. Gas damping must be minimized to achieve high sensitivity of MEMS, such as resonators. In contrast, high damping may be preferable in some MEMS to mitigate their shock response and transient performance. There is a great need for accurate, yet computationally inexpensive, theoretical models to predict squeeze-film gas damping ratios in MEMS especially at low gas pressures in which many MEMS are packaged.

Many theoretical models exist to predict squeeze-film gas damping ratios in MEMS and they can be classified into two groups according to the squeeze-film gas force derivation procedure [5]: (a) Reynolds-equation-based continuum models [6-12] and (b) non-gradient-based sub-continuum models [13-16].

In the former, the squeeze-film gas force is derived from the Reynolds equation, which has been widely used in fluid film lubrication [17-22]. The latter category of models is based on the collisions of gas molecules impinging on the surfaces of a moving structure. Some researchers argue that 
the Reynolds-equation-based approach is not valid because continuum models cannot consider gas rarefaction effects in a very low ambient pressure regime [16, 23]. However, recent works by Sumali [5], Veijola [10] and Nayfeh and Younis [7] showed that theoretical continuum models could accurately predict the squeeze-film damping characteristics of microstructures even at low ambient pressures, corresponding to higher Knudsen numbers $(K n)$. Also, Gallis and Torczynski [11] suggested modified pressure boundary conditions by using direct simulation Monte Carlo (DSMC) to increase the accuracy of the Reynolds-equation-based model.

More recently, Sumali [5] performed a series of rigorous experiments using a rigid microplate suspended by four folded beam springs over a wide range of Knudsen numbers $\left(K n \cong 10^{-2}-10^{3}\right)$ and showed that Veijola's compact model [10] provided a superior performance over all other continuum models and in fact the predictions were as good as those from Gallis and Torczynski [11]. Veijola theoretically limited his compact model to low Knudsen numbers $(0.01 \leqslant$ $K n \leqslant 0.1)$ in the slip regime [10], and yet surprisingly Sumali's recent results showed that Veijola's compact model [10] accurately predicts the gas damping well into the free molecular regime (moderate to high Knudsen numbers). The underlying physics behind this result is not yet completely understood. These recent studies suggest that Reynoldsequation-based continuum models that take into account gas rarefaction effects such as Veijola's compact model [10] are computationally inexpensive and may be capable of predicting the gas damping ratios of MEMS even at low ambient pressures. Veijola's compact model [10] was derived for rigid oscillating structures, and a theoretical extension and experimental validation of this model to flexible oscillating structures with multiple eigenmodes at a high Knudsen number regime have not yet been made although the effect of structural flexibility using Veijola's older model [6] has been studied by Pandey and Pratap [8]. Likewise Gallis and Torczynski's [11] slip-jump model was derived for a crosssection rigidly oscillating near a wall, and its extensions to flexible microcantilevers and the corresponding experimental validation have not been performed.

In this work, we extend Veijola's compact model [10] and Gallis and Torczynski's Navier-Stokes slip-jump model [11] to flexible microcantilever structures and predict the gas damping ratios of the higher order bending modes from low to moderately high Knudsen numbers and validate them experimentally. Two closed-form expressions of frequency response functions for a flexible microcantilever beam affected by the squeeze-film phenomenon are presented to predict the gas damping ratios of multiple bending modes: the first is derived for an inertially excited microcantilever beam and the second one is derived for a microcantilever beam excited by an external force, for example an electrostatic force. To validate the theoretical models, we carefully carried out experiments on poly-Si microcantilever beams at various ambient pressures (0.001-83.6 Torr). In addition, this paper presents a comprehensive review of prior work on Reynoldsequation-based continuum models for gas damping in MEMS and discusses the influence of the gas rarefaction coefficient,

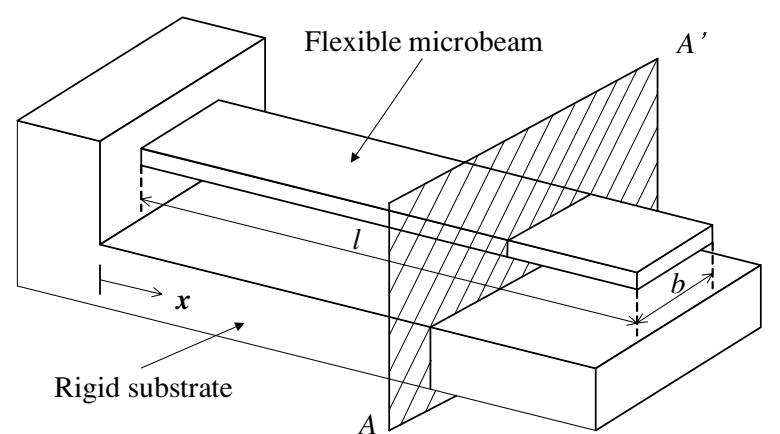

(a)

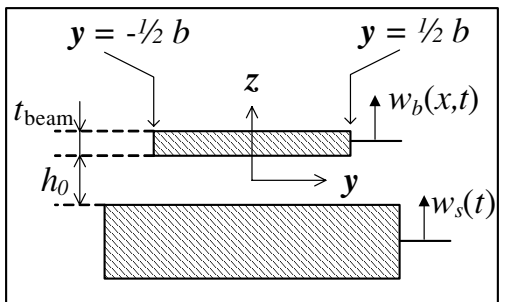

(b)

Figure 1. (a) A schematic of the microcantilever of length $l$, width $b$ and thickness $t_{\text {beam }}$ under squeeze-film effect and $(b)$ cross-section view $\left(A A^{\prime}\right)$ of the microcantilever vibrating close to a rigid substrate.

gap height and pressure boundary condition assumptions on squeeze-film gas forces.

\section{Review of the literature on continuum models for gas damping in MEMS}

By way of background to the present work, it is useful to present a fairly detailed summary of other numerous Reynolds-equation-based continuum models for MEMS. The well-known Reynolds equation governing the fluid flow in gas films is developed from the Navier-Stokes equation and the continuity equation, assuming that the gap of the gas film is much smaller than the surface dimensions [17-22]. The general Reynolds equation used in MEMS is linearized on the assumption that the displacement amplitude of the microstructure is very small [24]. The linearized Reynolds equation under an isothermal process assumption for squeezefilm fluid under a flexible microcantilever beam of length $l$, width $b$ and thickness $t_{\text {beam }}$ in figure 1 can be written in a dimensionless form as

$$
\left(\frac{b^{2}}{l^{2}} \frac{\partial^{2} \bar{p}}{\partial \bar{x}^{2}}+\frac{\partial^{2} \bar{p}}{\partial \bar{y}^{2}}\right)-\alpha^{2} \frac{\partial \bar{p}}{\partial t}=\alpha^{2} \frac{\partial \bar{w}_{b}}{\partial t}, \quad \alpha^{2}=\frac{12 G \cdot b^{2}}{h_{0}^{2} p_{a}},
$$

where $\bar{x}$ and $\bar{y}$ denote non-dimensional $x$ and $y$ coordinates, respectively, and $\bar{p}$ is defined as the ratio of small pressure variation $p$ to ambient pressure $p_{a}: \bar{x}=x / l, \bar{y}=y / b, \bar{p}=$ $p / p_{a}$. The symbol $\bar{w}_{b}$ denotes the normalized displacement of the microcantilever beam in the $z$ direction: $\bar{w}_{b}=$ $w_{b} / h_{0}$, where $w_{b}$ is the relative vertical displacement of the microcantilever with respect to the rigid substrate and $h_{0}$ is a nominal gap height between the microcantilever beam and the substrate. The symbol $G$ is the gas rarefaction coefficient. 
Table 1. Assumptions made to derive the gas force term in the Reynolds-equation-based approaches.

\begin{tabular}{|c|c|c|c|}
\hline Reference & Gas rarefaction effect $(G)$ & Pressure boundary condition & Gas compressibility $\left(\sigma^{\mathrm{g}}\right)$ \\
\hline Veijola et al [6] & $\mu_{1}^{\mathrm{a}}$ & $\bar{p}=0^{\mathrm{e}}$ & $\sigma \Varangle 1$ \\
\hline Nayfeh and Younis [7] & $\mu_{1}$ & $\bar{p}=0$ at open end $\nabla \bar{p}=0^{\mathrm{f}}$ at closed end & $\sigma \Varangle 1$ \\
\hline Pandey and Pratap [8] & $\mu_{1}$ & $\bar{p}=0$ at open end $\nabla \bar{p}=0$ at closed end & $\sigma \Varangle 1$ \\
\hline Veijola et al [9] & $\mu_{2}{ }^{b}$ & $\bar{p}=0$ considering a bordering effect & $\sigma \ll 1$ \\
\hline Veijola [10] & $\mu_{3}{ }^{\mathrm{c}}$ & $\bar{p}=0$ & $\sigma \Varangle 1$ \\
\hline Gallis and Torczynski [11] & $\mu^{\mathrm{d}}$ & Free from the boundary condition & $\sigma \ll 1$ \\
\hline Pandey et al [12] & $\mu$ & Partially blocked boundaries & $\sigma \ll 1$ \\
\hline Blech [21] & $\mu$ & $\bar{p}=0$ & $\sigma \Varangle 1$ \\
\hline
\end{tabular}

${ }^{\mathrm{a}} \mu_{1}=\frac{\mu}{1+9.638 \cdot \mathrm{Kn}^{1.159}}$.

${ }^{\mathrm{b}} \mu_{2}=\frac{\mu}{1+6 \sigma_{p} \cdot K n}$.

${ }^{\mathrm{c}} \mu_{3}=\mu / Q_{\mathrm{pr}}, Q_{\mathrm{pr}}$ is a relative flow rate coefficient including gas inertial effect [10].

d $\mu$ is a nominal viscous coefficient.

e $\bar{p}=p / p_{a}, \bar{p}=0$ (trivial pressure boundary condition).

${ }^{\mathrm{f}} \nabla \bar{p}=0$ (trivial flow boundary condition).

${ }^{\mathrm{g}} \sigma=\alpha^{2} \omega$ is the squeeze number: $\alpha^{2}=12 G \cdot b^{2} /\left(h_{0}^{2} p_{a}\right)$.

In order to simplify equation (1), various assumptions have been made as follows.

(a) Consider gas rarefaction effects in three ways depending on the effective viscous coefficient expression. The first way is to use the old effective viscous coefficient $\mu_{1}$, which is expressed only as a function of the Knudsen number $K n=\lambda / h_{0}$, where $\lambda$ is the mean free path of a gas molecule and $h_{0}$ is the gap height between two plates [6-8]:

$$
\mu_{1}=\frac{\mu}{1+9.638 \cdot K n^{1.159}},
$$

where $\mu$ is a nominal viscous coefficient. The second way is to use another expression for the effective viscous coefficient $\mu_{2}$, a function of $K n$ and slip coefficient $\sigma_{p}$, by applying the slip velocity boundary condition directly [9]:

$$
\mu_{2}=\frac{\mu}{1+6 \sigma_{p} \cdot K n} .
$$

The third method includes the slip boundary condition and inertial effects of the gas to derive a new effective viscous coefficient $\mu_{3}$, which is a function of $K n, \sigma_{p}$ and angular frequency $\omega$ :

$$
\mu_{3}=\mu / Q_{\mathrm{pr}},
$$

where $Q_{\mathrm{pr}}$ is the relative flow rate coefficient containing $\sigma_{p}$ and $\omega[10]$. In theoretical models for very low Knudsen number regimes, the gas rarefaction coefficient $G$ was replaced by the normal viscous coefficient $\mu[12,21]$.

(b) Impose trivial pressure boundary conditions $(\bar{p}=0)$ on the borders of the fluid analysis region in most theoretical models (see figures 1( $a$ ) and $(b)$ ). However, Nayfeh and Younis [7] and Pandey and Pratap [8] applied a trivial flow boundary condition $(\nabla \bar{p}=0)$ to the closed edge at which no flow is permitted. More recently, Veijola et al [9] attempted to decrease errors due to the trivial pressure boundary conditions by including elongation of surface dimensions. Pandey et al [12] classified the boundary conditions on the side of a vibrating microstructure into three kinds: fully closed boundary, fully open boundary and partially open boundary $\left(\delta_{1} \cdot \bar{p}+\delta_{2} \cdot \nabla \bar{p}=0\right)$, where $\delta_{1}$ and $\delta_{2}$ are constant values depending on the closed and open portions of the boundaries, respectively. They used a fully open boundary condition considering the extension of the original length to increase the accuracy of theoretical damping ratios of a MEMS torsion mirror where the air gap is comparable to the structural length. Gallis and Torczynski [11] developed a squeeze-film gas force which is free from the pressure boundary condition by using DSMC.

(c) Ignore or include gas compressibility depending on the squeeze number. The squeeze number $\sigma=\alpha^{2} \omega$ determines the gas compressibility in the Reynolds equation. The dimensionless number is proportional to angular frequency $\omega$ but inversely proportional to ambient pressure $p_{\mathrm{a}}$. If $\sigma \ll 1$, the gas in continuum models can be assumed to be incompressible and the third term on the left-hand side in equation (1) can be ignored. Otherwise, the time-dependent pressure term cannot be ignored. The only previous work $[9,12]$ focused on pressure boundary conditions assumed that the gas is incompressible.

Table 1 summarizes the assumptions made about gas rarefaction effects, pressure boundary condition and gas compressibility. Furthermore, table 2 compares the microstructures, effective Knudsen number regime and validation method of previous theoretical continuum models.

\section{Squeeze film-damping force}

In this work, we will focus on using two recently improved Reynolds-equation-based theories, namely (a) Veijola's compact model [10] and (b) Gallis and Torczynski's slip-jump model [11], and these are described below.

\subsection{Veijola's compact model [10]}

We develop the squeeze-film gas force applied to a long, slender and flexible microcantilever in figure 1 from the modified Reynolds equation derived by Veijola [10], who used 
Table 2. Structure, effective $K n$ range and validation method of continuum models suggested to describe the squeeze-film damping phenomenon.

\begin{tabular}{|c|c|c|c|}
\hline Reference & Structure & Effective $K n$ range $^{\mathrm{b}}$ & Validation method \\
\hline Veijola et al [6] & Rigid plate & $4.3-194.7$ & Experiment \\
\hline Nayfeh and Younis [7] & Flexible double clamped plate & $32.6-1086300$ & Experiment (first bending mode) [30] \\
\hline Pandey and Pratap [8] & Flexible cantilever plate & 0.0451 & $\begin{array}{l}\text { Experiment and simulation (the first } \\
\text { three bending modes) }\end{array}$ \\
\hline Veijola et al [9] & Rigid plate & $0.0167-0.128$ & Commercial package simulation (Elemer) \\
\hline Veijola [10] & Rigid plate & $0.01-1000$ & Experiment by Sumali [5] \\
\hline Gallis and Torczynski [11] & Rigid plate & $0.0085-1.16$ & Commercial package simulation \\
\hline Pandey et al [12] & Rigid plate & $0.00008-0.003$ & Commercial package simulation (ANSYS) \\
\hline Andrews et $a l^{\mathrm{a}}[25]$ & Rigid plate & $0.0042-64$ & Experiment \\
\hline Cheng and Fang [27] & Rigid plate & $0.038-1442$ & Experiment \\
\hline Bao et al [28] & Perforated rigid plate & Not specified & Commercial package simulation (ANSYS) \\
\hline Pandey et al [29] & Perforated rigid/flexible plate & 0.0182 & Commercial package simulation (ANSYS) \\
\hline
\end{tabular}

a Andrews et al used the effective viscous coefficient proposed by Dushman and Lafferty [26].

$\mathrm{b}$ The effective Knudsen number regime was provided by previous papers or calculated for the specific values in those papers.

$\mu_{3}$ in equation (4) reflecting the slip boundary condition and gas inertia to consider the gas rarefaction effect:

$\mu_{3}=\mu / Q_{\mathrm{pr}}$,

$Q_{\mathrm{pr}}=\frac{12 \mu}{\mathrm{j} \omega \rho h_{o}^{3} q}\left[\frac{q h_{o}-\left(2-q^{2} \sigma_{p} \lambda h_{o}\right) \tanh \left(q h_{o} / 2\right)}{1+\sigma_{p} \lambda q \tanh \left(q h_{o} / 2\right)}\right]$,

where $q=\sqrt{\mathrm{j} \omega \rho / \mu}$ is the complex frequency variable, $\rho$ is the fluid density and $\mathrm{j}=\sqrt{-1}$. The diffuse scattering model is used for the slip boundary condition $\left(\sigma_{p}=1.016\right)$ and the mean free path $\lambda$ of a gas molecule is calculated by the following equation $[10,31]$ :

$$
\lambda=\frac{\mu}{p_{a}} \sqrt{2 \bar{R} T}
$$

where $\bar{R}$ is the individual gas constant and $T$ is the temperature: $\bar{R}=286.9 \mathrm{~J} \mathrm{~K}^{-1} \mathrm{~kg}^{-1}$ for air and $T=298 \mathrm{~K}$. The length of the beam is assumed to greatly exceed its nominal width, and trivial pressure boundary conditions are imposed on both ends of the cross-section $y= \pm \frac{1}{2} b$ in figure $1(b)$.

Since the velocity field varies slowly along the length of the beam in comparison with variations across its width for $l \gg b$ [32], the first term on the left-hand side of equation (1) can be neglected: $(b / l)^{2} \partial^{2} \bar{p} / \partial \bar{x}^{2} \approx 0$. Taking the Fourier transform of equation (1) and using trivial pressure boundary conditions $(\bar{p}=0)$ at both sides of the microcantilever beam in figure $1(b)$ results in

$$
P(\bar{x}, \bar{y} \mid \omega)=p_{a}\left(\frac{\cosh (\beta \cdot \bar{y})}{\cosh \left(\frac{1}{2} \beta\right)}-1\right) \cdot W_{b}(\bar{x} \mid \omega),
$$

where capital letters represent the angular frequency dependence of variables [32]: $P(\bar{x}, \bar{y} \mid \omega)=\int_{-\infty}^{\infty} p(\bar{x}, \bar{y} \mid t) \cdot \mathrm{e}^{\mathrm{j} \omega t} \mathrm{~d} t$ and $W_{b}(\bar{x} \mid \omega)=\int_{-\infty}^{\infty} \bar{w}_{b}(\bar{x} \mid t) \cdot \mathrm{e}^{\mathrm{j} \omega t} \mathrm{~d} t$. The symbol $\beta$ is a function of the complex squeeze number $\sigma_{c}$ :

$$
\begin{gathered}
\beta=\sqrt{\mathrm{j} \sigma_{c}}, \\
\sigma_{c}=\frac{12 \mu b^{2}}{Q_{\mathrm{pr}} p_{a} h_{0}^{2}} \omega .
\end{gathered}
$$

The Fourier-transformed force per unit length $F_{d}$ applied to the cross-section of the microcantilever in figure $1(b)$ is

$$
F_{d}(\bar{x} \mid \omega)=-b \int_{-1 / 2}^{1 / 2} P(\bar{x}, \bar{y} \mid \omega) \mathrm{d} \bar{y}=-b \cdot P_{\text {gas }}^{\mathrm{V}}(\omega) \cdot W_{b}(\bar{x} \mid \omega),
$$

where $P_{\text {gas }}^{\mathrm{V}}$ is the pressure variation due to the squeeze-film phenomenon that Veijola assumed [10] and consists of imaginary and real components, which contribute inertial/elastic restoring force and damping force, respectively:

$$
P_{\text {gas }}^{\mathrm{V}}(\omega)=p_{a}\left(\frac{2}{\beta} \tanh (\beta / 2)-1\right) .
$$

\subsection{Gallis and Torczynski's model [11]}

Another squeeze-film gas force is derived from the improved Reynolds equation (equation (12)) with a modified form of the boundary condition (equation (13)) suggested by Gallis and Torczynski [18], who assumed that the gas motion is quasistatic and isothermal from the following Reynolds equation:

$$
\begin{gathered}
\frac{\partial \bar{w}_{b}}{\partial t}=\frac{h_{0}^{2} p_{a}}{12 \mu b^{2}}\left(1+\chi \frac{6 \Lambda}{h_{o}}\right)\left(\frac{\partial^{2} \bar{p}}{\partial \bar{y}^{2}}+\frac{b^{2}}{l^{2}} \frac{\partial^{2} \bar{p}}{\partial \bar{x}^{2}}\right), \\
\bar{p}=-\eta \cdot \frac{h_{0}}{b} \frac{\partial \bar{p}}{\partial \bar{y}}-\gamma\left(\frac{12 \mu}{p_{a}} \frac{\partial \bar{w}_{b}}{\partial t}\right)\left(1+\chi \frac{6 \Lambda}{h_{o}}\right)^{-1},
\end{gathered}
$$

where the slip length $\Lambda$ is equal to the mean free path $\lambda$ of a gas molecule in the diffuse scattering model and the symbols of $\eta$, $\gamma, \chi$ are coefficients, which are determined through empirical correlations with DSMC simulation:

$$
\begin{gathered}
\chi=\frac{1+8.834 \cdot\left(\Lambda / h_{0}\right)}{1+5.118 \cdot\left(\Lambda / h_{0}\right)}, \\
\eta=\frac{0.634+1.572 \cdot\left(\Lambda / h_{0}\right)}{1+0.537 \cdot\left(\Lambda / h_{0}\right)}, \\
\gamma=\frac{0.445+11.20 \cdot\left(\Lambda / h_{0}\right)}{1+5.510 \cdot\left(\Lambda / h_{0}\right)} .
\end{gathered}
$$




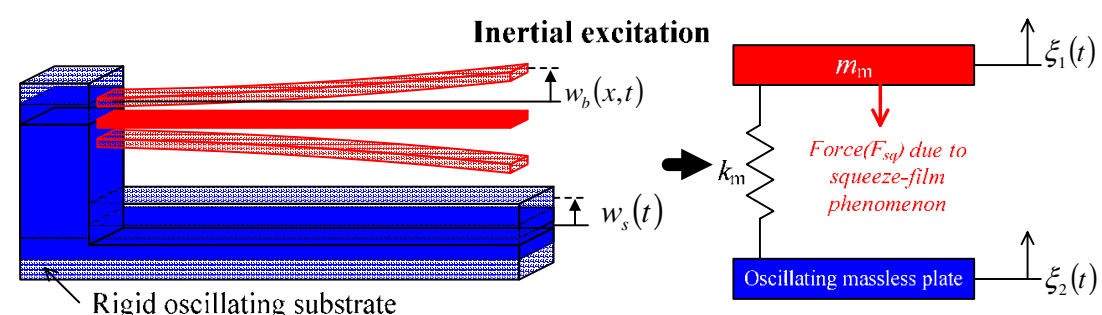

(a)

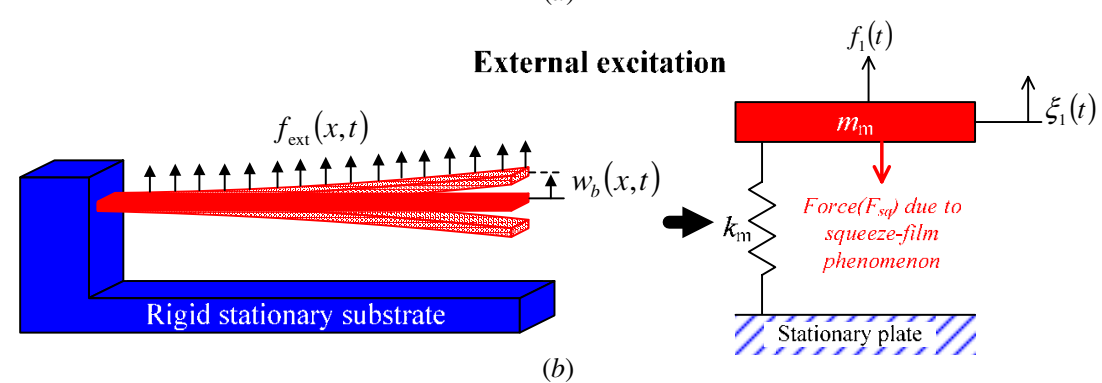

Figure 2. Flexible microcantilevers and harmonic mechanical systems used for frequency response functions and curve-fitting equations: (a) inertial excitation and $(b)$ external excitation.

The squeeze-film gas force $f_{d}$ per unit length is

$$
f_{d}(\bar{x} \mid t)=-b \int_{-1 / 2}^{1 / 2} \bar{p}(\bar{x}, \bar{y}) \mathrm{d} \bar{y} .
$$

Taking the Fourier transform into both sides of equation (15) yields

$$
\begin{gathered}
F_{d}(\bar{x} \mid \omega)=-b \cdot P_{\mathrm{gas}}^{\mathrm{GT}} \cdot W_{b}(\bar{x} \mid \omega), \\
P_{\mathrm{gas}}^{\mathrm{GT}}=\mathrm{j} \omega \cdot\left(\frac{b}{h_{0}}\right)^{2} \cdot \mu \cdot\left(1+\chi \frac{6 \Lambda}{h_{0}}\right)^{-1} \\
\times\left(1+3 \eta\left(\frac{h_{0}}{b}\right)+3 \gamma\left(\frac{2 h_{0}}{b}\right)^{2}\right),
\end{gathered}
$$

where pressure variation $P_{\text {gas }}^{\mathrm{GT}}$ has only an imaginary term. Compared with Veijola's model, Gallis and Torczynski's model neglects inertial and elastic forces in the squeeze film.

\section{Frequency response functions of a flexible microcantilever}

Before using these two models to predict the damping ratios of flexible cantilevers, it is important to understand the dependence of the frequency response on the form of external excitation. Two typical frequency response functions for a microcantilever vibrating near a rigid substrate are derived with different excitation methods. The first one represents the frequency characteristics of an inertially excited flexible microcantilever beam shown in figure 2(a) as might happen for cantilever resonators used for small mass detection. The second one is developed for an externally excited flexible microcantilever beam shown in figure $2(b)$ and can be used for an electrostatically actuated RF switch or magnetically excited atomic force microscope (AFM) cantilever beam.

Since the squeeze-film phenomenon happens due to the small change in gap height between the microcantilever and the substrate, the squeeze-film gas force can be derived in the same way, independent of the excitation method. The gap $h(x, t)$ and the relative displacement $w_{b}(x, t)$ with respect to the displacement $w_{s}(t)$ of a rigid substrate are related by $h(x, t)=w_{b}(x, t)+h_{0}$. The cantilever displacement $w_{b}(x, t)$ is assumed to be much less than the nominal gap height $h_{0}$ for linear vibration analysis.

\subsection{Frequency response function of an inertially excited flexible microcantilever beam}

In the first theoretical model (figure 2(a)), the displacement $w_{s}$ of the rigid oscillating substrate and the relative displacement $w_{b}$ of the microcantilever beam satisfy the following simple beam vibration equation [33]:

$$
\frac{\mathrm{EI} \cdot h_{0}}{l^{4}} \frac{\partial^{4} \bar{w}_{b}}{\partial \bar{x}^{4}}+m h_{0} \frac{\partial^{2}}{\partial t^{2}}\left(\bar{w}_{s}+\bar{w}_{b}\right)=f_{d},
$$

where EI is the flexural rigidity and $\bar{w}_{s}=w_{s} / h_{0}$. The symbol $m$ is the mass per unit length of the beam and $m=\rho_{\text {beam }} \cdot S$, where $\rho_{\text {beam }}$ and $S$ are the mass density and the crosssectional area of the beam, respectively. The symbol $f_{d}(\bar{x}, t)$ denotes a squeeze-film gas force per unit length applied to the microcantilever beam. Taking the Fourier transform of equation (18) yields

$$
\begin{aligned}
& \frac{\mathrm{EI} \cdot h_{0}}{l^{4}} \frac{\mathrm{d}^{4} W_{b}(\bar{x} \mid \omega)}{\mathrm{d} \bar{x}^{4}}-m h_{0} \omega^{2}\left(W_{s}(\omega)+W_{b}(\bar{x} \mid \omega)\right) \\
& \quad=F_{d}(\bar{x} \mid \omega),
\end{aligned}
$$

where $W_{s}(\omega)=\int_{-\infty}^{\infty} \bar{w}_{s}(t) \cdot \mathrm{e}^{\mathrm{j} \omega t} \mathrm{~d} t$. Substituting equation (10) or (16) for $F_{d}(\bar{x} \mid \omega)$ in equation (19) yields $\frac{\mathrm{EI} \cdot h_{0}}{l^{4}} \frac{\mathrm{d}^{4} W_{b}}{\mathrm{~d} \bar{x}^{4}}-m h_{0} \omega^{2} \cdot W_{b}+b \cdot P_{\text {gas }} \cdot W_{b}=m h_{0} \omega^{2} \cdot W_{s}$,

where $P_{\text {gas }}$ becomes $P_{\text {gas }}^{\mathrm{V}}$ (equation (11)) in the extended Veijola's model and $P_{\text {gas }}^{\mathrm{GT}}$ (equation (17)) in the extended Gallis and Torczynski's model. 
Consider the eigenmodes $\Phi_{n}(x)$ of a cantilever beam satisfying the simple beam vibration equation

$$
\frac{\mathrm{d}^{4} \Phi_{n}}{\mathrm{~d} \bar{x}^{4}}-\beta_{n}^{4} \Phi_{n}=0, \quad \beta_{n}^{4}=\frac{m l^{4}}{\mathrm{EI}} \omega_{n}^{2},
$$

where $\omega_{n}$ is the $n$th eigenfrequency, which is obtained from the characteristic equation (22) and each eigenmode is normalized satisfying the condition in equation (23) [34]:

$$
\begin{gathered}
\cos \beta_{n} \cosh \beta_{n}+1=0 \\
\int_{0}^{1} \beta_{n}^{4} \Phi_{n}(\bar{x}) \cdot \Phi_{m}(\bar{x}) \mathrm{d} \bar{x}=\delta_{m n} .
\end{gathered}
$$

Substituting $W_{b}(\bar{x} \mid \omega)=\sum A_{n}(\omega) \Phi_{n}(\bar{x})$ into equation (20) and using the orthogonality of eigenmodes, the ratio of the $n$th participation factor $A_{n}$ to the displacement $W_{s}$ of a rigid oscillating substrate is obtained:

$$
\begin{aligned}
\frac{A_{n}}{W_{s}}= & \frac{\omega^{2} / \omega_{n}^{2}}{1-\omega^{2} / \omega_{n}^{2}+b / h_{0} \cdot P_{\text {gas }} / \beta_{n}^{4} \cdot l^{4} / \mathrm{EI}} \\
& \times \beta_{n}^{4} \int_{0}^{1} \Phi_{n}(\eta) \mathrm{d} \eta .
\end{aligned}
$$

Finally, the frequency response function $H_{1}(\bar{x} \mid \omega)$ of the inertially excited microcantilever beam is obtained as follows:

$$
H_{1}(\bar{x} \mid \omega)=\frac{W_{b}(\bar{x} \mid \omega)}{W_{s}(\omega)}=\sum_{n=1} \frac{A_{n}(\omega)}{W_{s}(\omega)} \Phi_{n}(\bar{x}) .
$$

\subsection{Frequency response function of an externally excited flexible microcantilever beam}

In the second theoretical model (figure 2(b)), the rigid substrate is stationary $\left(w_{s}=0\right)$ and an external uniform force $f_{\text {ext }}$ is applied to the microcantilever, whose movement creates the squeeze-film gas force $f_{d}$. The non-dimensional transverse displacement $\bar{w}_{b}$ of a flexible microcantilever beam satisfies the following partial differential equation [34]:

$$
\frac{\mathrm{EI} \cdot h_{0}}{l^{4}} \frac{\partial^{4} \bar{w}_{b}}{\partial \bar{x}^{4}}+m h_{0} \frac{\partial^{2} \bar{w}_{b}}{\partial t^{2}}=f_{d}+f_{\mathrm{ext}} .
$$

In a similar way as in the previous section, taking the Fourier transform and substituting equation (10) or (16) yields

$$
\begin{aligned}
& \frac{\mathrm{EI} \cdot h_{0}}{l^{4}} \frac{\mathrm{d}^{4} W_{b}(\bar{x} \mid \omega)}{\mathrm{d} \bar{x}^{4}}-m h_{0} \omega^{2} W_{b}(\bar{x} \mid \omega)+b \cdot P_{\text {gas }} \cdot W_{b}(\bar{x} \mid \omega) \\
& =F_{\text {ext }}(\omega),
\end{aligned}
$$

where $F_{\text {ext }}(\omega)=\int_{-\infty}^{\infty} f_{\text {ext }}(t) \cdot \mathrm{e}^{\mathrm{j} \omega t} \mathrm{~d} t$. Applying the mode superposition method to equation (27), the ratio of the $n$th participating factor $A_{n}$ to the external force $F_{\text {ext }}$ is obtained:

$$
\begin{aligned}
\frac{A_{n}}{F_{\mathrm{ext}}} & =\frac{1 / \omega_{n}^{2}}{1-\omega^{2} / \omega_{n}^{2}+b / h_{0} \cdot P_{\mathrm{gas}} / \beta_{n}^{4} \cdot l^{4} / \mathrm{EI}} \\
& \cdot \frac{\beta_{n}^{4}}{m \cdot h_{0}} \cdot \int_{0}^{1} \Phi_{n}(\eta) \mathrm{d} \eta .
\end{aligned}
$$

Therefore, the frequency response function $H_{2}(\bar{x} \mid \omega)$ of an externally excited flexible microcantilever beam can be expressed as follows:

$$
H_{2}(\bar{x} \mid \omega)=\frac{W_{b}(\bar{x} \mid \omega)}{F_{\mathrm{ext}}(\omega)}=\sum_{n=1} \frac{A_{n}(\omega)}{F_{\mathrm{ext}}(\omega)} \Phi_{n}(\bar{x}) .
$$

4.3. Comparison of damping ratios obtained by the two different excitation methods

Prior experimental efforts $[5,6,8,27,30]$ to measure the squeeze-film damping ratios of suspended microstructures do not distinguish the difference between the excitation mechanisms used to extract the damping ratios or $Q$ factors of different eigenmodes. Here, we show that there are subtle differences in the modal damping ratios extracted using different excitation mechanisms and different extraction methods [35]. To this end, we compare two commonly used damping data extraction methods as follows.

(a) The first method is where theoretical transfer functions in equations (25) and (29) are compared with the frequency response functions (30) and (31) derived for a single eigenmode. The specific values of the dimensions of microcantilever beam 5 in table 3 were used to simulate the theoretical transfer functions using the extended Veijola's model. Specifically, the simulated transfer functions are fitted to the curve-fitting equations $\left(H_{1}^{f}(\omega)\right.$ and $\left.H_{2}^{f}(\omega)\right)$ of the simple harmonically excited models in figures 2(a) and $(b)$, respectively:

$$
H_{1}^{f}(\omega)=\frac{\left(\omega / \omega_{r}\right)^{2}}{\sqrt{\left(1-\left(\omega / \omega_{r}\right)^{2}\right)^{2}+\left(2 \zeta_{r} \cdot \omega / \omega_{r}\right)^{2}}} \cdot A
$$

for the model in figure $2(a)$,

$$
H_{2}^{f}(\omega)=\frac{1}{\sqrt{\left(1-\left(\omega / \omega_{r}\right)^{2}\right)^{2}+\left(2 \zeta_{r} \cdot \omega / \omega_{r}\right)^{2}}} \cdot A
$$

for the model in figure $2(b)$,

where $\omega_{r}$ and $\zeta_{r}$ are the natural frequency and damping ratio of each resonant mode, respectively. The physical meaning of $A$ is specified and the curve-fitting equation derivation procedure is described in detail in the appendix. The three parameters $\omega_{r}, \zeta_{r}, A$ are determined through the curve-fitting method.

(b) The second method is where the damping ratios are extracted using the half-power point method, which is valid only for a one-degree-of-freedom mass-springdamping system applied by an external force when the damping ratio is much less than ' 0.05 ' [36].

Figure 3 compares the two frequency response functions with the different excitation methods at the end point: figure 3(a) shows the magnitudes of the two frequency response functions around the first bending mode frequency at an ambient pressure of 298 mTorr and figure 3(b) shows those at an ambient pressure of 25.1 Torr. The magnitude at each frequency is normalized by the peak magnitude to make a fair comparison between the two frequency response functions. Since the two frequency response functions are almost the same at low ambient pressure as shown in figure $3(a)$, the calculated damping ratios from equations (30) and (31) are equal to that calculated by the half-power point method. As shown in figure $3(b)$, in contrast, the difference of two frequency response functions at a high ambient pressure gives different theoretical damping ratios. The difference in the damping ratio $\left(\zeta_{r}\right)$ of the first bending mode calculated by 
Table 3. Specific values of variables used to calculate squeeze-film damping ratios.

\begin{tabular}{|c|c|c|c|c|}
\hline \multirow[b]{2}{*}{ Symbol } & \multirow{2}{*}{\multicolumn{2}{|c|}{ Quantity }} & \multicolumn{2}{|c|}{ Value } \\
\hline & & & Microcantilever beam & Microcantilever beam 5 \\
\hline$b$ & $\begin{array}{r}\text { Widt } \\
\text { microcanti }\end{array}$ & $\begin{array}{l}\text { of a } \\
\text { ver beam }{ }^{a}\end{array}$ & \multicolumn{2}{|c|}{$18 \mu \mathrm{m}$} \\
\hline$l$ & $\begin{array}{r}\text { Leng } \\
\text { microcanti }\end{array}$ & $\begin{array}{l}\text { of a } \\
\text { ver beam }\end{array}$ & $300 \mu \mathrm{m}$ & $500 \mu \mathrm{m}$ \\
\hline$h_{o}$ & Gap height ${ }^{\mathrm{b}}$ & $\begin{array}{l}\text { Maximum } \\
\text { Mean } \\
\text { Minimum }\end{array}$ & $\begin{array}{l}1.93 \mu \mathrm{m} \\
1.83 \mu \mathrm{m} \\
1.73 \mu \mathrm{m}\end{array}$ & $\begin{array}{l}1.74 \mu \mathrm{m} \\
1.40 \mu \mathrm{m} \\
1.04 \mu \mathrm{m}\end{array}$ \\
\hline$t_{\text {beam }}$ & \multicolumn{2}{|c|}{$\begin{array}{l}\text { Thickness of a } \\
\text { microcantilever beam }\end{array}$} & \multicolumn{2}{|c|}{$2.25 \mu \mathrm{m}$} \\
\hline E & \multicolumn{2}{|c|}{$\begin{array}{l}\text { Young's modulus of a } \\
\text { microcantilever beam }{ }^{\text {a }}\end{array}$} & \multicolumn{2}{|c|}{$160 \mathrm{GPa}$} \\
\hline$\rho_{\text {beam }}$ & \multicolumn{2}{|c|}{$\begin{array}{c}\text { Density of a } \\
\text { microcantilever beam }\end{array}$} & \multicolumn{2}{|c|}{$2330 \mathrm{~kg} \mathrm{~m}^{-3}$} \\
\hline$\mu$ & \multicolumn{2}{|c|}{$\begin{array}{c}\text { Nominal viscous } \\
\text { coefficient }\end{array}$} & \multicolumn{2}{|c|}{$18.6 \mu \mathrm{Pa} \mathrm{s}$} \\
\hline$\rho$ & \multicolumn{2}{|c|}{ Density of air } & \multicolumn{2}{|c|}{$1.21 \mathrm{~kg} \mathrm{~m}^{-3}$} \\
\hline
\end{tabular}

a The width, length, thickness, density and Young's modulus of microcantilever beams were provided by Sandia National Lab and their effectiveness is validated by comparing theoretical bending mode frequencies and experimental resonant frequency at $1 \mathrm{mTorr}$ : the maximum tolerances of microcantilever beams 3 and 5 are $0.2 \%$ and $1.2 \%$, respectively.

$\mathrm{b}$ The gap height was optically measured by using white light interferometry and monotonically decreased from the clamped end to the free end.

using equations (30) and (31) was 2.7\%: $\zeta_{r}$ is 0.2567 for $H_{1}$ and 0.2498 for $H_{2}$. Also, the damping ratios calculated by the half-power-point method are 0.3691 and 0.2887 , respectively. Since the damping ratio calculated by the half-power-point method can have some error as the ambient pressure increases, a physics-based curve-fitting equation depending on the excitation method should be used to extract an exact damping ratio. In what follows, we will extract damping ratios from inertially excited frequency response functions by fitting the measured response to equation (30).

\section{Experiment}

In order to validate our theoretical approach, careful experiments were carried out on the cantilever array discovery platform (CADP) fabricated at the Sandia National Labs. The CADP chip was fabricated out of poly-silicon through the Bosch etching method, which is known to improve uniformity for advanced MEMS applications. The chip has many suspended microcantilever beams of varying lengths to accommodate a wide variety of experiments: composite beams for thin film testing; scanning probe beams with heaters, resistance thermometers and magnetic particles; beams with optical gratings for interferometry and torsional beams. In this experiment, as shown in figure 4 , microcantilever beams of varying lengths above a substrate were used. The averaged gap heights are $1.40 \mu \mathrm{m}$ and $1.83 \mu \mathrm{m}$ for microcantilever beams 5 and 3, respectively. One end of each microcantilever beam is anchored to the rigid substrate and the other end is free.

Since the distance between two adjacent microcantilevers is comparable to their width, the fluid or structure coupling with neighboring microcantilevers can affect experimental results. To avoid this potential problem, all the beams neighboring a cantilever of interest were broken off in each case, as shown in figure $4(b)$. The prepared CADP was placed in a vacuum chamber with air as shown in figure 5. The ambient pressure in the vacuum chamber (MMR Inc.'s variable temperature micro probe vacuum chamber) was changed from 83.6 Torr down to five orders of magnitude lower with the vacuum pump.

The flexible microcantilever beam is excited by a piezoelectric shaker with a pseudo-random signal. The magnitude of the input signal fed to the piezoelectric shaker was adjusted so that the maximum transverse displacement of the cantilever beams was less than $10 \mathrm{~nm}$, which is at least two orders of magnitude smaller than the gap height, thus ensuring linear gas damping. In our experiments, only an inertial excitation method is used for microcantilever beams 3 and 5 . Laser Doppler vibrometry (Polytec's MSA-400) with two laser beams measured velocity signals at two points simultaneously. The position of one laser beam was fixed and used to measure the vibration at one point on the oscillating rigid substrate. The other laser beam scanned the microcantilever beam: the number of measuring points is 33 for microcantilever beam 3 and 39 for microcantilever beam 5 .

Two kinds of experimental methods were used to acquire accurate experimental data and save measuring time.

(1) At high ambient pressures (142 mTorr-83.6 Torr), frequency response functions were measured. To increase the accuracy of experimental gas damping ratios, the frequency resolution was adjusted depending on ambient pressure and all measured frequency response functions were averaged ten times: the finest frequency resolution was $2.5 \mathrm{~Hz}$

(2) In contrast, ring-down data were measured at low ambient pressures (1-70 mTorr) because the damping ratios are 


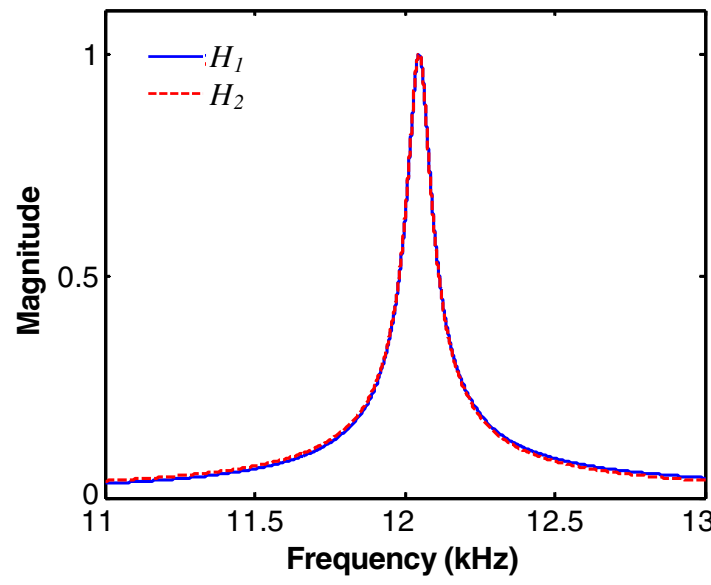

(a)

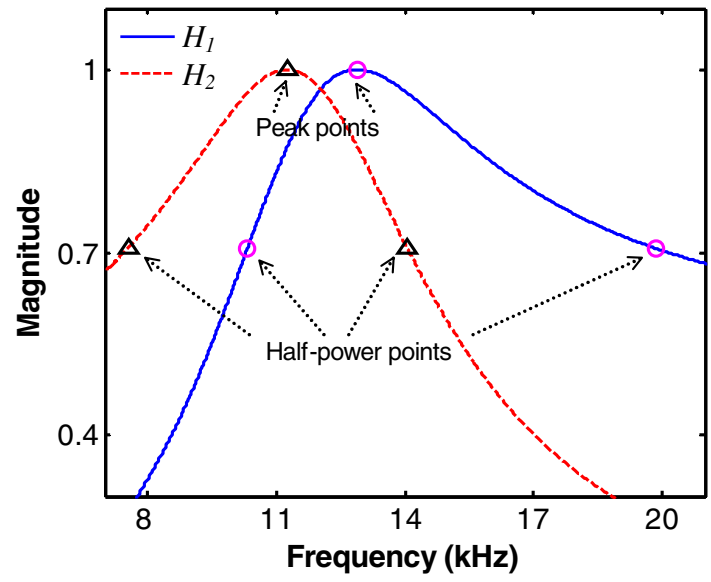

(b)

Figure 3. Comparison of frequency response functions of an inertially excited microcantilever beam and an externally excited microcantilever beam at (a) 289 mTorr and (b) 25.1 Torr: $\bigcirc$ and $\triangle$ represent three points for the half-power point method in two frequency response functions, respectively. These are theoretical predictions using the extended Veijola's model and parameter values (microcantilever beam 5 with a mean value of the gap height) given in table 3 .

extremely low and a $3 \mathrm{~dB}$ bandwidth is too small in the frequency response function to acquire enough fine frequency resolution around peak frequencies. The ringdown data were acquired as follows: first, a selected resonant mode of a microcantilever beam was driven at an associated resonant frequency by a piezoelectric shaker; second, the input signal was turned off; and then the decaying vibration signal of a microcantilever beam was acquired in the time domain. At each ambient pressure, the ring-down data were separately measured five times and the mean value of calculated damping ratios is used as a representative damping ratio.

\subsection{Experimental squeeze-film damping ratio calculation}

Two different curve-fitting methods are used in order to calculate the gas damping ratios of three bending modes of a microcantilever beam from the measured frequency response functions and ring-down data. While the curve-fitting

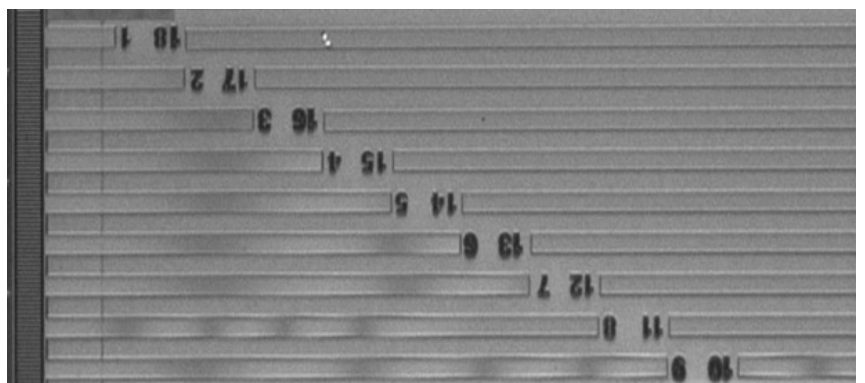

(a)

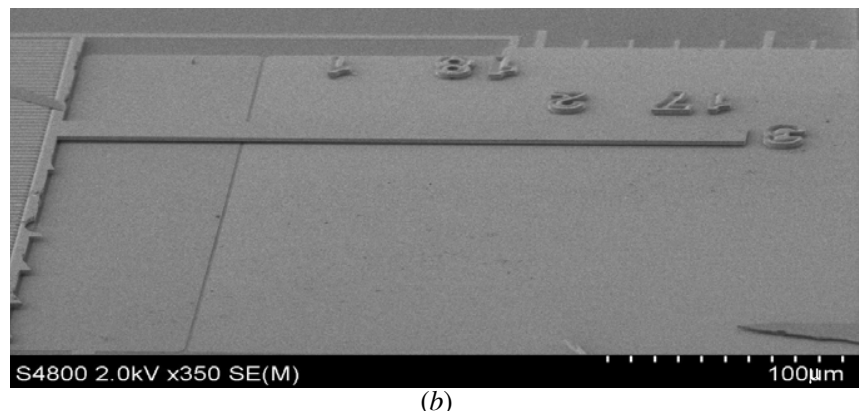

Figure 4. Cantilever array discovery platform: all of the other cantilevers were broken except for one cantilever in each experiment. (a) Complete array and $(b)$ tested cantilever.

equation (30) is used for frequency response functions measured at higher ambient pressures, the logarithmic decrement method with the Hilbert transform [37] is used for low ambient pressures where the ring-down data are acquired.

Figure 6(a) shows the frequency response function for the third bending mode of the flexible microcantilever beam 5 at $p_{a}=20.2$ Torr. The measured frequency response function $H_{m}(\omega)$ is converted to the gap transmission function $H_{g}(\omega)$ for calculating squeeze-film damping ratios [5]:

$$
H_{g}(\omega)=H_{m}(\omega)-1 \text {. }
$$

From the converted frequency response functions at six different points around the end of each microcantilever beam as shown in figure $6(b)$, the mean, maximum and minimum values of the gas damping ratios are calculated.

Figure 6(c) shows the ring-down data of microcantilever beam 5 measured after being driven at the first bending mode frequency at 5 mTorr. Ring-down data were acquired five times at point 2 in figure $6(b)$ at each ambient pressure, and linear damping components were extracted by the logarithmic decrement method with the Hilbert transform [37], which has already been proven in previous work [5]. The minimum, mean and maximum values of damping ratio $\zeta$ at each ambient pressure were calculated.

\subsection{Subtraction of structural damping}

The contribution of structural damping, or non-squeeze-film damping, was subtracted by following the method suggested by Sumali [5]. The rationale of the subtraction method is that the structural damping ratio must be excluded in the experimental damping ratios at low ambient pressures because it is comparable to the gas damping ratio. Figure 7 shows the total damping ratios of the first bending mode 


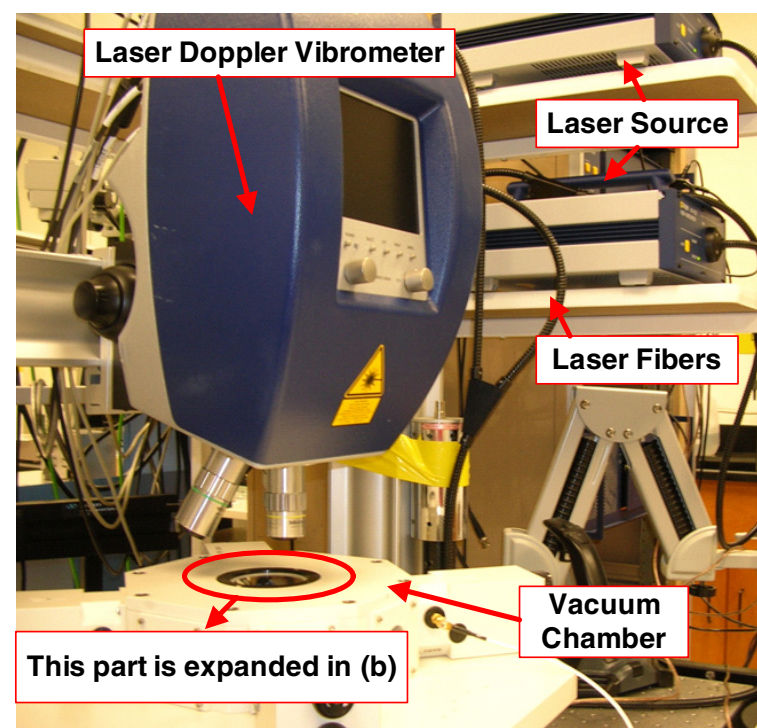

(a)

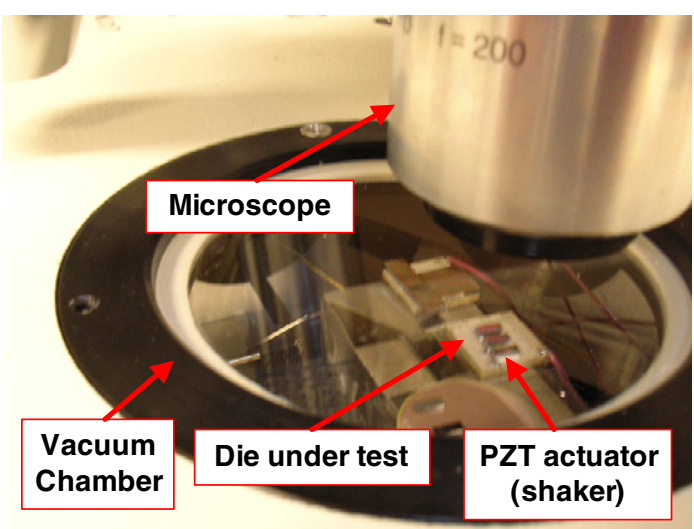

(b)

Figure 5. Experimental setup to acquire vibration signals of a flexible microcantilever beam at various ambient pressures: $(a)$ laser vibrometer and vacuum chamber, $(b)$ microscopic lens and die under test.

of microcantilever beam 3, which were obtained from experimental data at the lowest five ambient pressures. In the ambient pressure range of the plot, the relationship between the damping ratio and ambient pressure can be assumed to be linear. That is, the damping ratio at zero ambient pressure is the structural damping ratio and is obtained by extrapolation with a linear-fitting equation.

\subsection{Comparison of experimental and theoretical squeeze-film damping ratios}

The nominal thickness, length and width of the microcantilever beams and a measured gap height listed in table 3 were used to calculate theoretical squeeze-film damping ratios. The three bending mode frequencies of microcantilever beams 3 and 5 measured at $1 \mathrm{~m}$ Torr were very close to theoretical natural frequencies with a tolerance of less than $0.2 \%$ and $1.2 \%$, respectively. The gap height between a microcantilever beam and a substrate was optically measured by using white light interferometry and monotonically decreased from the clamped

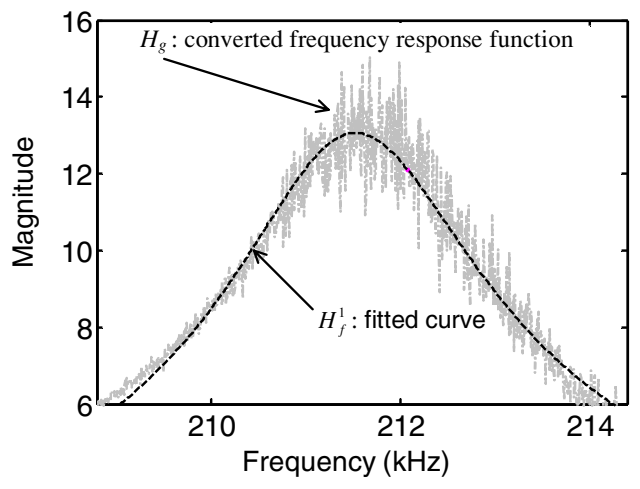

(a)

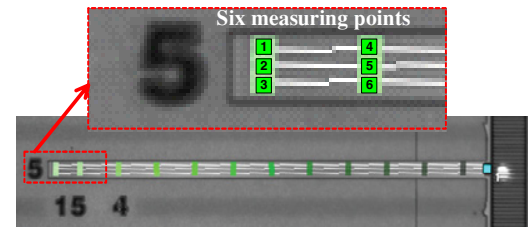

(b)

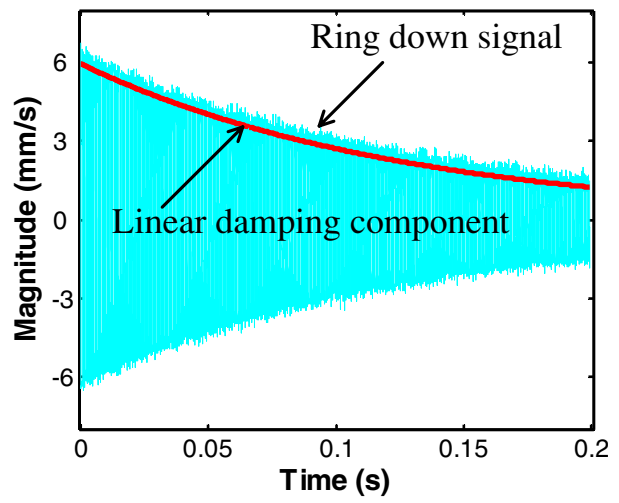

(c)

Figure 6. (a) Frequency response function $\left(H_{g}\right)$ and its fitted curve $\left(H_{f}^{1}\right)$ of the third bending mode of microcantilever beam 5 at 20.2 Torr, $(b)$ measuring points and $(c)$ ring-down data for the first bending mode of microcantilever beam 5 at 5 mTorr.

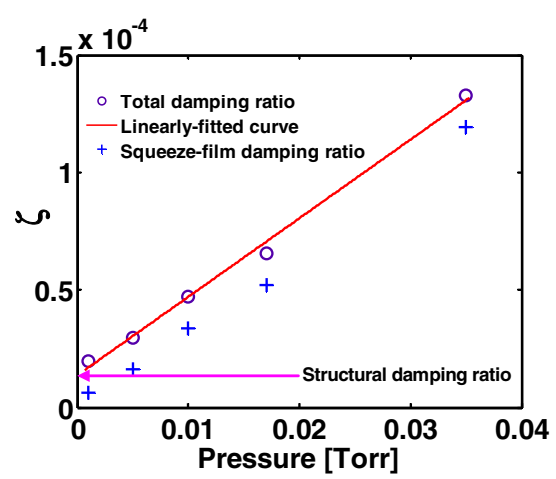

Figure 7. Structural damping ratio extraction in the first bending mode of microcantilever beam 3 .

end to the free end. The minimum, mean and maximum values of the gap height are listed in table 3 .

Figure 8 compares theoretical and experimental squeezefilm damping ratios of the first three bending modes of microcantilever beam 5 over a wide range of ambient 

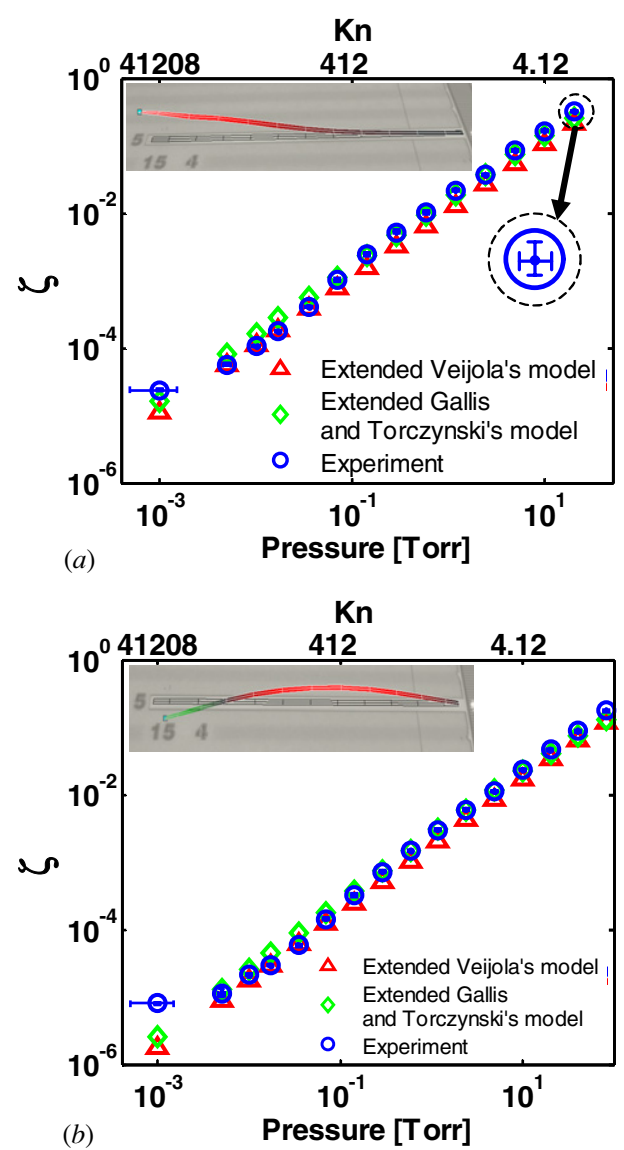

$\mathrm{Kn}$

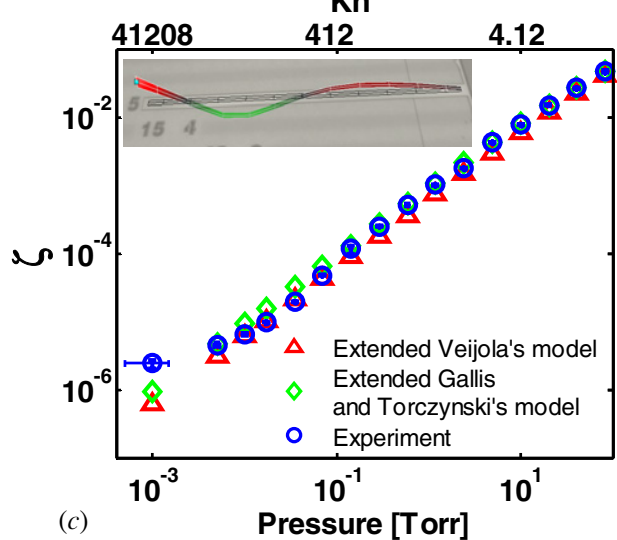

Figure 8. Comparison of squeeze-film damping ratios obtained theoretically and experimentally for microcantilever beam 5: $(a)$ first bending mode, $(b)$ second bending mode and $(c)$ third bending mode. Experimental damping ratios have two error bars: vertical error bar represents the maximum and minimum values of the damping ratio at each ambient pressure and horizontal error bar represents the effective pressure range of the controlled ambient pressure of a vacuum chamber. Squeeze-film damping ratios of two extended models were calculated for the mean value of the gap height in table 3.

pressures. The extended Gallis and Torczynski's model predicts well squeeze-film gas damping ratios at high ambient pressures (142 mTorr-83.6 Torr) with a maximum error of $25.94 \%$. In contrast, the damping ratios calculated by the extended Veijola's model are much closer to experimental damping ratios than the extended Gallis and Torczynski's model at low ambient pressures (2-70 mTorr). The experimental damping ratio at each ambient pressure is plotted with a solid circle representing an averaged damping ratio, a vertical error bar showing the maximum and minimum gas damping ratios and a horizontal error bar showing the effective ambient pressure range. The gas damping ratios predicted by the two extended theoretical models were calculated and plotted for the mean value of the gap height.

Figure 9 compares the theoretical and experimental squeeze-film damping ratios of the first three bending modes of microcantilever beam 3. The two extended models qualitatively predict well the squeeze-film damping ratios of the microcantilever beam, but the differences between theoretical and experimental results increased as ambient pressure increased unlike those of microcantilever 5. The reason is that the length-to-width ratio of microcantilever 3 is smaller than that of microcantilever 5. In the theoretical analysis (section 3), we assumed $l \gg b$ to restrict fluid motion in the cantilever's cross-sectional plane. As the length-towidth ratio decreases, this assumption may break down.

\section{Discussion}

Clearly, the flexible microcantilever beam extension of the gas damping model suggested by Gallis and Torczynski generally performs better than that of Veijola's compact model at high ambient pressures. At low ambient pressures, however, the extension of Veijola's compact model to flexible beams also does quite well. Here we examine the underlying uncertainties in gas damping ratio prediction using these models. The uncertainties in Veijola's extension considered in this investigation are due to (a) gap height, (b) gas rarefaction coefficient and (c) pressure boundary condition. In the extension of Gallis and Torczynski's model, the main uncertainty lies in the knowledge of gap height. In what follows, we will focus on predictions versus experimental data for microcantilever beam 5 .

First, consider uncertainties due to (a) gap height, (b) gas rarefaction coefficient and (c) pressure boundary condition in the extended Veijola's model for the second bending mode of microcantilever beam 5. The first two uncertainties are shown in figure $10(a)$. Such analyses have been carried out for all eigenmodes of microcantilever beams 3 and 5, and here we chose to discuss one representative result. The maximum and minimum gap heights used in this calculation are derived from real gap height measurements using white light interferometry (as discussed in section 5) and are listed in table 3. The green error bars reflecting uncertainty in the assumption on the gas rarefaction effect are based on the maximum and minimum predictions using the different model equations (2)-(4). These theoretical predictions assume the trivial pressure boundary condition $(\bar{p}=0)$ at $y= \pm \frac{1}{2} b$. Note that when the trivial flow boundary condition $(\nabla \bar{p}=0)$ is assumed at $y= \pm \frac{1}{2} b$, the predicted gas damping ratios vanish, which has already been proven in a previous work by Darling et al [38]. This modeling uncertainty is not plotted on the graph. The real boundary condition for open ends at both ends $y= \pm \frac{1}{2} b$ may be assumed to be located somewhere between two extreme 

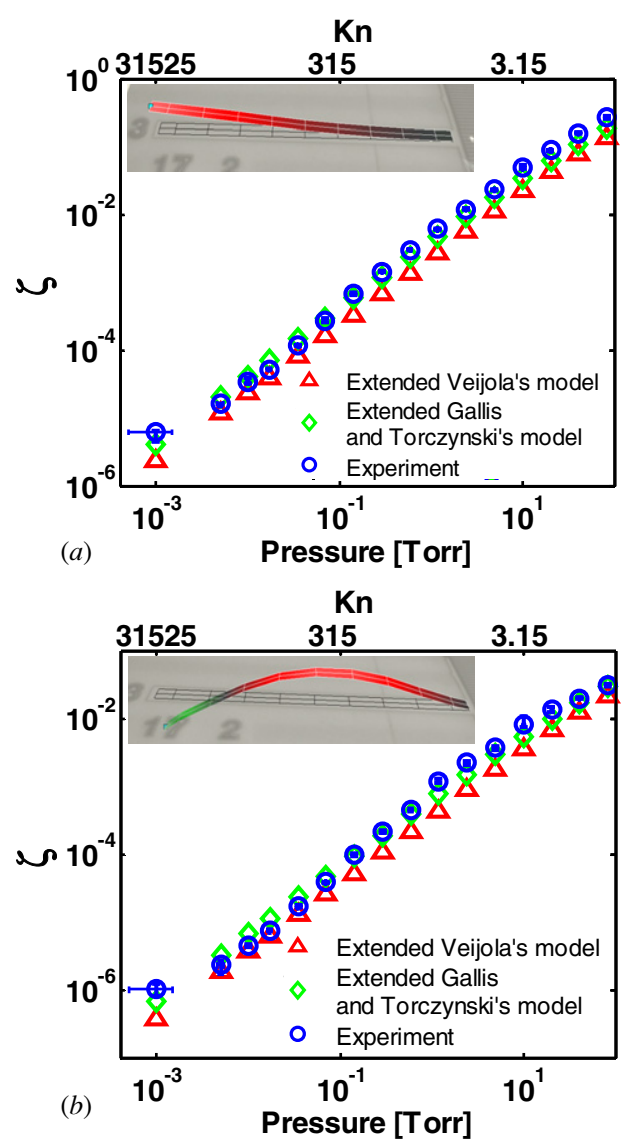

$\mathrm{Kn}$

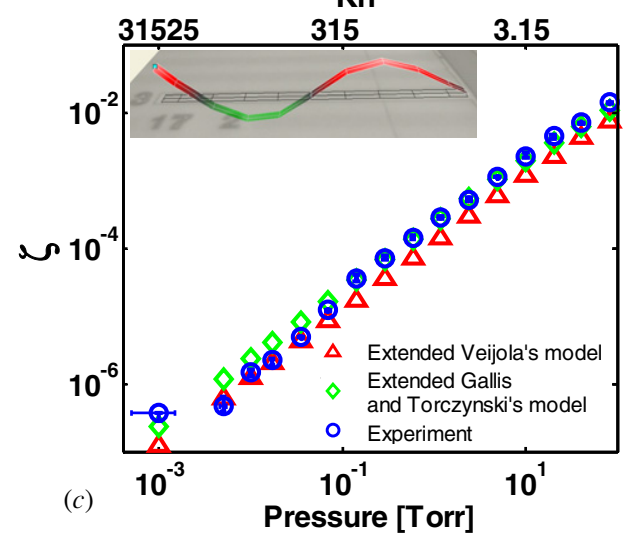

Figure 9. Comparison of squeeze-film damping ratios obtained theoretically and experimentally for microcantilever beam 3: $(a)$ first bending mode, $(b)$ second bending mode and $(c)$ third bending mode. Experimental damping ratios have two error bars: vertical error bar represents the maximum and minimum values of the damping ratio at each ambient pressure and horizontal error bar represents the effective pressure range of the controlled ambient pressure of a vacuum chamber. Squeeze-film damping ratios of two extended models were calculated for the mean value of the gap height in table 3.

boundary conditions, the trivial pressure boundary condition $(\bar{p}=0)$ and the trivial flow boundary condition $(\nabla \bar{p}=0)$. A small error incurred when using the trivial pressure boundary condition results from the small difference between the forces per unit length, as in equations (10), (16), (19), developed by the subsequent pressure distributions obtained using the
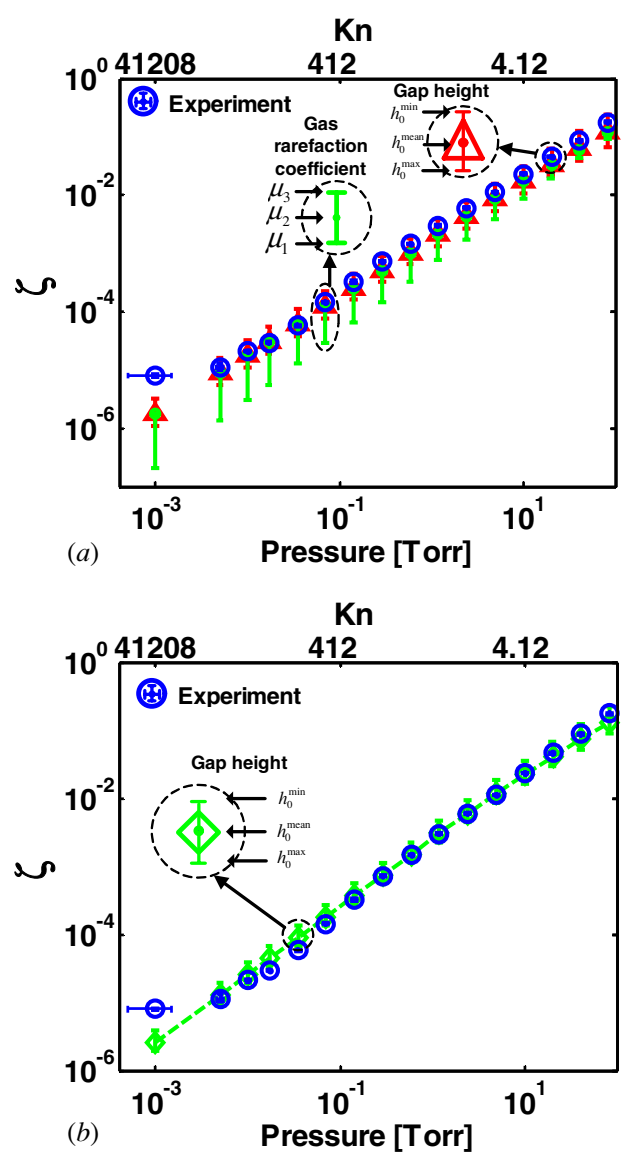

Figure 10. Squeeze-film damping ratios of the second bending mode of microcantilever beam 5 including uncertainties of $(a)$ the extended Veijola's model and $(b)$ the extended Gallis and Torczynski's model which have two uncertainties and one uncertainty, respectively. To consider uncertainty in the gap height, the damping ratios were calculated for the minimum, mean and maximum values of the gap height in table 3 . To compare gas rarefaction assumptions, the damping ratios were calculated for $\mu_{1}$ in equation (2), $\mu_{2}$ in equation (3) and $\mu_{3}$ in equation (4). The theoretical predictions assume the trivial pressure boundary condition $(\bar{p}=0)$ at $y= \pm \frac{1}{2} b$ in the extended Veijola's model.

trivial pressure boundary condition and by the actual pressure distribution across the cross-section [39].

Based on figure $10(a)$, it is clear that while the gas rarefaction model uncertainty (modeling uncertainty) and the gap uncertainty (geometric uncertainty) lead to significant variability in gas damping ratio prediction, by far the greatest uncertainty in Veijola's compact model is a modeling uncertainty due to the pressure boundary condition. It must be noted that in Veijola's compact model, the choice of the trivial pressure boundary condition is not based on any rational approximation, but is simply chosen since a boundary condition assumption needs to be made to derive the gas damping force. It is for this reason that we refer to this as a model uncertainty.

Second, we consider uncertainty due to the gap height in the extended Gallis and Torczynski's model for the second bending mode of microcantilever beam 5 in figure $10(b)$. For the maximum and minimum gap heights listed in table 3 , squeeze-film gas damping ratios were calculated and compared 
with experimental results. Since the modified pressure boundary condition in their original model is derived from DSMC and reflects the gas rarefaction effect, the uncertainty due to the pressure boundary condition and gas rarefaction coefficient is not considered in this figure.

As shown in figure $10(b)$, squeeze-film damping ratios calculated at minimum or maximum gap height increase the prediction accuracy of the extended Gallis and Torczynski's model except for a $1 \mathrm{mTorr}$ damping ratio. The experimental damping ratios fall within the limits predicted by Gallis and Torczynski's model if the uncertainty due to the gap height is considered. However, we do not argue that the theoretical model used is robust so as to be applicable to all squeeze-film damping ratio predictions for MEMS. It is desirable that the gap height variation is regarded as an important factor to be carefully dealt with in the prediction of squeeze-film damping ratios of flexible MEMS.

Gap height variation can be included in deriving frequency response functions in section 4 , but it requires numerical integration and matrix inversion. Replacing $h_{0}$ by $h_{0}(x)$ in equation (20) and following the same procedure as in section 4.1, the following matrix form is obtained:

$$
\begin{aligned}
{[\mathbf{M}+} & \mathbf{C}+\mathbf{K}]\{\mathbf{A}\}=\mathrm{EI} / l^{4} \cdot \omega^{2} \cdot W_{s}\{\mathbf{F}\}, \\
\mathbf{M}= & {\left[M_{n n}\right], \quad M_{n n}=\left(1-\omega^{2} / \omega_{n}^{2}\right) \cdot \mathrm{EI} / l^{4}, } \\
\mathbf{C}= & {\left[C_{n m}\right], \quad C_{n m}=\mathrm{j} \cdot b \cdot \operatorname{Im}\left(P_{\text {gas }}\right) } \\
& \times \int_{0}^{1} 1 / h_{0}(\bar{x}) \cdot \Phi_{n}(\bar{x}) \cdot \Phi_{m}(\bar{x}) \mathrm{d} \bar{x}, \\
\mathbf{K}= & {\left[K_{n m}\right] K_{n m}=b \cdot \operatorname{Re}\left(P_{\text {gas }}\right) } \\
& \times \int_{0}^{1} 1 / h_{0}(\bar{x}) \cdot \Phi_{n}(\bar{x}) \cdot \Phi_{m}(\bar{x}) \mathrm{d} \bar{x},
\end{aligned}
$$

where $\mathbf{M}$ is the diagonal matrix, but $\mathbf{C}$ and $\mathbf{K}$ have off-diagonal terms of the type $\int_{0}^{1} 1 / h_{0}(\bar{x}) \cdot \Phi_{n}(\bar{x}) \cdot \Phi_{m}(\bar{x}) \mathrm{d} \bar{x} \neq 0$. Thus, the non-uniform gap causes a coupling between previously uncoupled cantilever eigenmodes. The symbols $\operatorname{Re}\left\{P_{\text {gas }}\right\}$ and $\operatorname{Im}\left\{P_{\text {gas }}\right\}$ represent the real and imaginary parts of $P_{\text {gas }}$, respectively: $P_{\text {gas }}=\operatorname{Re}\left\{P_{\text {gas }}\right\}+\mathrm{j} \cdot \operatorname{Im}\left\{P_{\text {gas }}\right\}$. Furthermore,

$$
\{\mathbf{F}\}=\left\{F_{1}, \ldots, F_{n}, \ldots, F_{N}\right\}^{T}, \quad F_{n}=\beta_{n}^{4} / \omega_{n}^{2} \cdot \int_{0}^{1} \Phi_{n}(\bar{x}) \mathrm{d} \bar{x}
$$

$\{\mathbf{A}\}=\left\{A_{1}, \ldots, A_{n}, \ldots, A_{N}\right\}^{T}$.

In order to obtain $A_{n}(\omega) / W_{s}(\omega)$ in equation (25), matrix inversion should be performed in equation (33). This approach is computationally expensive. Therefore, it is more efficient to use an average gap height value and include gap uncertainties based on measured gap variations (maximum and minimum gap heights) instead of the exact gap height profile for each cantilever beam.

\section{Conclusions}

This paper presented a new improved Reynolds-equationbased theoretical model to predict squeeze-film damping ratios of flexible microcantilever beams vibrating close to a rigid substrate. Recent models [10, 11] were extended to include the flexibility of the microcantilever beams and were experimentally validated for higher order bending modes even in very low ambient pressure regimes. In the validation experiment, ring-down data and frequency response functions were measured to increase the accuracy of the measured data and reduce the required experimental time, depending on the ambient pressure. We investigated the uncertainties in damping ratio prediction introduced due to assumptions on the gas rarefaction effect, gap height and pressure boundary conditions.

This work makes three contributions to theoretical approaches in MEMS dynamics. First, our work is a first attempt to theoretically calculate squeeze-film damping ratios of higher order bending modes of flexible microcantilevers in high Knudsen number regimes. Second, the influence of the model and geometric uncertainties on the prediction of the gas damping ratio using Reynolds-equation-based methods will be helpful to future work on the design of flexible microstructures in MEMS. Third, careful experimental results were obtained to validate our theoretical models.

\section{Acknowledgments}

This material is based upon work supported by the Department of Energy (National Nuclear Security Administration) under Award Number DE-FC52-08NA28617 and by the Sandia National Labs under contract no. 623235. Part of this work was conducted at Sandia National Laboratories, Albuquerque, NM. Sandia is a multi-program laboratory operated under Sandia Corporation, a Lockheed Martin Company, for the United States Department of Energy under contract DE-AC0494-AL85000. The first author was also supported by the Korea Research Foundation Grant (KRF-2007-357-D00008) funded by the Korean Government (MOEHRD). The authors would like to thank Professor Alina Alexeenko and $\mathrm{PhD}$ student Xiaohui Guo of Purdue University for valuable discussions on this paper.

\section{Appendix}

In a simple inertially excited harmonic system (right-handside model in figure $2(a)$ ), the additional force due to the squeeze-film phenomenon is assumed to consist of inertia force, damping force and elastic force, which are proportional to the relative motion of the upper rigid plate with respect to the lower oscillating massless rigid plate as show in figure A1(a): the subscript 'sq' denotes squeeze film. The modal mass and modal stiffness of each bending mode are denoted by $m_{m}$ and $k_{m}$, respectively. The absolute displacements of the upper rigid plate and the lower rigid plate are denoted by $\xi_{1}(t)$ and $\xi_{2}(t)$, respectively. 


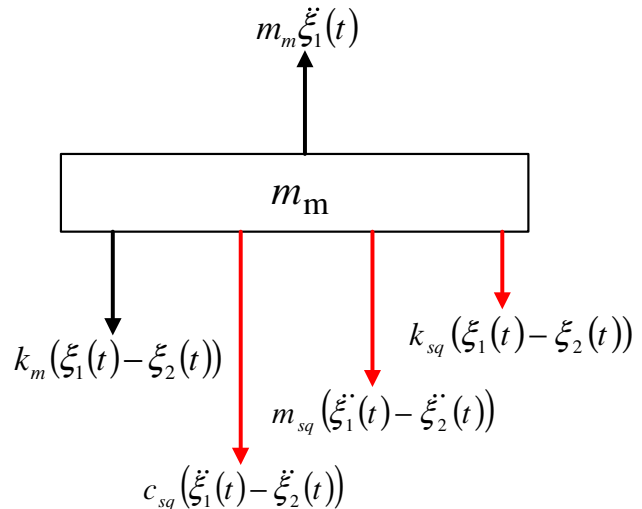

(a)

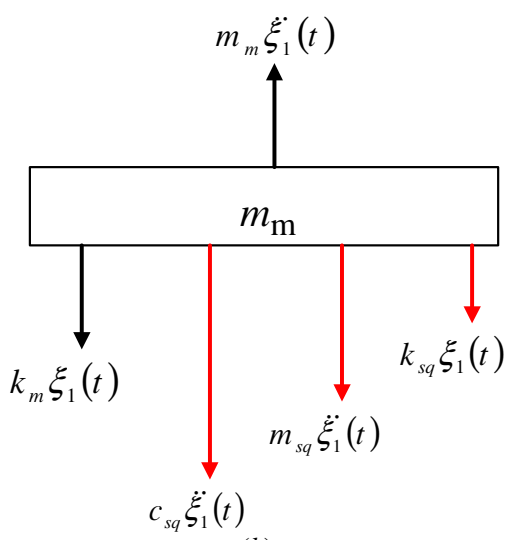

(b)

Figure A1. Free-body diagrams for the curve-fitting equations to express gap transmissibility of simple harmonic systems in figures $2(a)$ and $(b)$ : $(a)$ for inertial excitation and $(b)$ for external excitation.

The equation of motion for the simple inertially excited system is expressed as follows:

$$
\begin{aligned}
& m_{m} \ddot{\xi}_{1}+m_{\mathrm{sq}}\left(\ddot{\xi}_{1}-\ddot{\xi}_{2}\right)+c_{\mathrm{sq}}\left(\dot{\xi}_{1}-\dot{\xi}_{2}\right)+k_{\mathrm{sq}}\left(\xi_{1}-\xi_{2}\right) \\
& \quad+k_{m}\left(\xi_{1}-\xi_{2}\right)=0,
\end{aligned}
$$

where $m_{\mathrm{sq}}, c_{\mathrm{sq}}$ and $k_{\mathrm{sq}}$ denote the mass, damping coefficient and stiffness coefficients related to the squeeze-film gas force. Substituting $\xi_{3}=\xi_{1}-\xi_{2}$ and rearranging equation (A.1) yield

$$
m_{\mathrm{eq}} \ddot{\xi}_{3}+c_{\mathrm{sq}} \dot{\xi}_{3}+k_{\mathrm{eq}} \xi_{3}=-m_{m} \ddot{\xi}_{2},
$$

where $m_{\mathrm{eq}}=m_{m}+m_{\mathrm{sq}}$ and $k_{\mathrm{eq}}=k_{m}+k_{\mathrm{sq}}$. Assuming the harmonic motion of $\xi_{2}$ and $\xi_{3}: \xi_{2}=\Xi_{2} \cdot \mathrm{e}^{\mathrm{j} \omega t}$ and $\xi_{3}=\Xi_{3} \cdot \mathrm{e}^{\mathrm{j} \omega t}$, the following transfer function is derived:

$$
\frac{\Xi_{3}}{\Xi_{2}}=\frac{\left(\omega / \omega_{r}\right)^{2}}{1-\left(\omega / \omega_{r}\right)^{2}+\mathrm{j} 2 \zeta_{r} \cdot \omega / \omega_{r}} \cdot A,
$$

where $\omega_{r}$ is the natural frequency, $A$ is the mass ratio and $\zeta_{r}$ is the damping ratio: $\omega_{r}=\sqrt{k_{\text {eq }} / m_{\text {eq }}}, A=m_{m} / m_{\text {eq }}$ and $\zeta_{r}=c_{\mathrm{sq}} \cdot \omega_{r} /\left(2 k_{\mathrm{eq}}\right)$. Finally, the following curve-fitting equation is obtained:

$H_{1}^{f}(\omega)=\left|\frac{\Xi_{3}}{\Xi_{2}}\right|=\frac{\left(\omega / \omega_{r}\right)^{2}}{\sqrt{\left(1-\left(\omega / \omega_{r}\right)^{2}\right)^{2}+\left(2 \zeta_{r} \cdot \omega / \omega_{r}\right)^{2}}} \cdot A$.

In a simple externally excited harmonic system (righthand-side model in figure $2(b)$ ), the squeeze-film forces are proportional to the absolute motion of the upper rigid plate as shown in figure $\mathrm{A} 1(b)$ because the lower rigid plate is stationary. The equation of motion for the simple model is expressed as follows:

$$
m_{m} \ddot{\xi}_{1}+m_{\mathrm{sq}} \ddot{\xi}_{1}+c_{\mathrm{sq}} \dot{\xi}_{1}+k_{\mathrm{sq}} \xi_{1}+k_{m} \xi_{1}=f_{1} .
$$

Assuming the harmonic motion of $\xi_{1}$ and $f_{1}: \xi_{1}=\Xi_{1} \cdot \mathrm{e}^{\mathrm{j} \omega t}$ and $f_{1}=F_{1} \cdot \mathrm{e}^{\mathrm{j} \omega t}$, the following frequency response function is derived:

$$
\frac{\Xi_{1}}{F_{1}}=\frac{1}{1-\left(\omega / \omega_{r}\right)^{2}+\mathrm{j} 2 \zeta_{r} \cdot \omega / \omega_{r}} \cdot A,
$$

where $\omega_{r}$ is the natural frequency, $A$ is the mass ratio and $\zeta_{r}$ is the damping ratio: $\omega_{r}=\sqrt{k_{\text {eq }} / m_{\text {eq }}}, A=1 / k_{\text {eq }}$ and $\zeta_{r}=c_{\mathrm{sq}} \cdot \omega_{r} /\left(2 k_{\mathrm{eq}}\right)$. Finally, the following curve-fitting equation is obtained:

$H_{2}^{f}(\omega)=\left|\frac{\Xi_{1}}{F_{1}}\right|=\frac{1}{\sqrt{\left(1-\left(\omega / \omega_{r}\right)^{2}\right)^{2}+\left(2 \zeta_{r} \cdot \omega / \omega_{r}\right)^{2}}} \cdot A$.

\section{References}

[1] Sumali H, Massad J E, Czaplewski D A and Dyck C W 2007 Waveform design for pulse-and-hold electrostatic actuation in MEMS Sensors Actuators A 134 213-20

[2] Grandaldi A and Decuzzi P 2006 The dynamic response of resistive microswitches: switching time and bouncing $J$. Micromech. Microeng. 16 1108-15

[3] Guo Z J, Mcgruer N E and Adams G G 2007 Modeling, simulation and measurement of the dynamic performance of an ohmic contact, electrostatically actuated RF MEMS switch J. Micromech. Microeng. 17 1899-909

[4] Czaplewski D A, Dyck C W, Sumali H, Massad J E, Kuppers J D, Reines I, Cowan W D and Tigges C P 2006 A soft-landing waveform for actuation of a single-pole single-throw ohmic RF MEMS switch J. Microelectromech. Syst. 15 1586-94

[5] Sumali H 2007 Squeeze-film damping in the free molecular regime: model validation and measurement on a MEMS J. Micromech. Microeng. 17 2231-40

[6] Veijola T, Kuisma H, Lahdenperä J and Ryhänen T 1995 Equivalent-circuit model of the squeezed gas film in a silicon accelerometer Sensors Actuators A 48 239-48

[7] Nayfeh A H and Younis M I 2004 A new approach to the modeling and simulation of flexible microstructures under the effect of squeeze-film damping J. Micromech. Microeng. 14 170-81

[8] Pandey A K and Pratap R 2007 Effect of flexural modes on squeeze film damping in MEMS cantilever resonators J. Micromech. Microeng. 17 2475-84

[9] Veijola T, Pursula A and Raback P 2005 Extending the validity of squeezed-film damper models with elongations of surface dimensions J. Micromech. Microeng. 15 1624-36

[10] Veijola T 2004 Compact models for squeezed-film dampers with inertial and rarefied gas effects J. Micromech. Microeng. 14 1109-18

[11] Gallis M A and Torczynski J R 2004 An improved Reynolds-equation model for gas damping of microbeam motion J. Microelectromech. Syst. 13 653-9

[12] Pandey A K, Pratap R and Chau F S 2007 Influence of boundary conditions on the dynamic characteristics of squeeze films in MEMS devices J. Microelectromech. Syst. 16 893-903

[13] Kádár Z, Kindt W, Bossche A and Mollinger J 1996 Quality factor of torsional resonators in the low-pressure region Sensors Actuators A 53 299-303 
[14] Li B, Wu H, Zhu C and Liu J 1999 The theoretical analysis on damping characteristics of resonant microbeam in vacuum Sensors Actuators 77 191-4

[15] Bao M, Yang H, Yin H and Sun Y 2002 Energy transfer model for squeeze-film air damping in low vacuum J. Micromech. Microeng. 12 341-6

[16] Hutcherson S and Ye W 2004 On the squeeze-film damping of micro-resonators in the free-molecule regime J. Micromech. Microeng. 14 1726-33

[17] Langolis W E 1962 Isothermal squeeze films Q. Appl. Math. 20 131-50

[18] Griffin W S, Richardson H H and Yamanami S 1966 A study of fluid squeeze-film damping Trans. ASME D 88 451-6

[19] Gross W A, Matsch L A, Castelli V, Eshel A, Vohr J H and Wildmann M 1980 Fluid Film Lubrication (New York: Wiley) pp 29-69

[20] Shukla J B, Kumar S and Chandra P 1980 Generalized Reynolds equation with slip at bearing surfaces: multiple-layer lubrication theory Wear $60253-68$

[21] Blech J J 1983 On isothermal squeeze films J. Lubr. Technol. $105615-20$

[22] Hamrock B J 1994 Fundamentals of Fluid Film Lubrication (New York: McGraw-Hill) pp 117-65

[23] Bhiladvala R B and Wang Z J 2004 Effect of fluids on the Q factor and resonance frequency of oscillating micrometer and nanometer scale beams Phys. Rev. E 69036307

[24] Bao M and Yang H 2007 Squeeze film air damping in MEMS Sensors Actuators A 136 3-27

[25] Andrews M, Harris I and Turner G 1993 A comparison of squeeze-film theory with measurements on a microstructure Sensors Actuators A 36 79-87

[26] Dushman S and Lafferty J M 1962 Scientific Foundation of Vacuum Technique (New York: Wiley)

[27] Cheng C and Fang W 2005 Tuning the quality factor of bulk micromachined structures using squeezed-film damping Microsyst. Technol. 11 104-10
[28] Bao M, Yang H, Sun Y and French P J 2003 Modified Reynolds' equation and analytical analysis of squeeze-film air damping of perforated structures J. Micromech. Microeng. 13 795-800

[29] Pandey A K, Pratap R and Chau F S 2007 Analytical solution of the modified Reynolds equation for squeeze film damping in perforated MEMS structures Sensors Actuators A 135 839-48

[30] Legtenberg R and Tilmans H A 1994 Electrostatically driven vacuum-encapsulated polysilicon resonators: I. Design and fabrication Sensors Actuators A 45 57-66

[31] Sharipov F and Seleznev V 1998 Data on internal rarefied gas flows J. Phys. Chem. Ref. Data 27 657-706

[32] Sader J E 1998 Frequency response of cantilever beams immersed in viscous fluids with applications to the atomic force microscope J. Appl. Phys. 84 64-76

[33] Meirovitch L 1967 Analytical Methods in Vibrations (New York: Macmillan) pp 299-300

[34] Meirovitch L 1986 Elements of Vibration Analysis (Singapore: McGraw-Hill) pp 220-27

[35] Xu X and Raman A 2007 Comparative dynamics of magnetically, acoustically, and Brownian motion driven microcantilevers in liquids J. Appl. Phys. 102034303

[36] Rao S S 2004 Mechanical Vibrations (Englewood Cliffs, NJ: Prentice-Hall) pp 234-5

[37] Sumali H 2007 Measuring natural frequency and non-linear damping on oscillating micro plates Proc. 13th Int. Conf. on Experimental Mechanics (Alexandroupolis, Greece, 1-7 July) (CD-ROM)

[38] Darling R B, Hivick C and Xu J 1998 Compact analytical modeling of squeeze film damping with arbitrary venting conditions using a Green's function approach Sensors Actuators A 70 32-41

[39] Guo X and Alexeenko A 2009 Compact model of squeeze-film damping based on rarefied flow simulations J. Micromech. Microeng. 19 1-7 